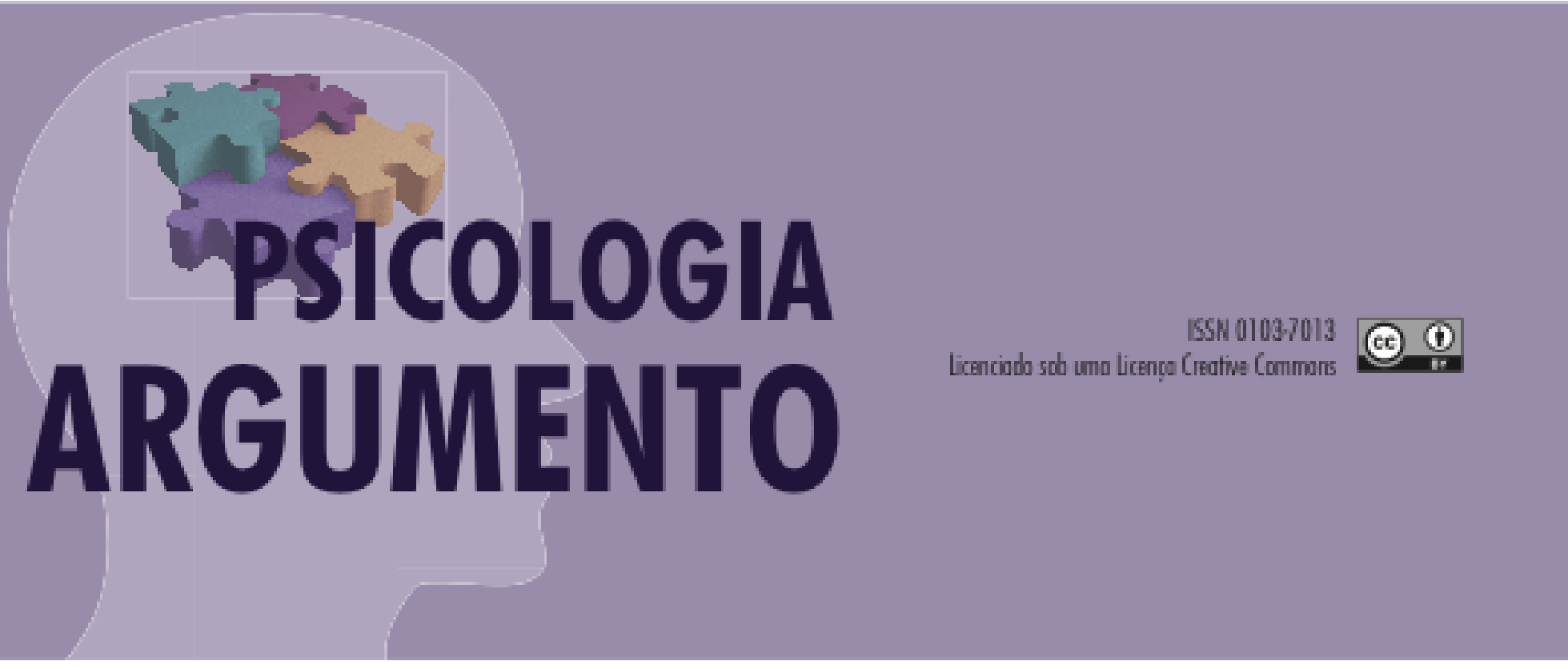

doi: 10.7213/psicol.argum.34.084.A007

\title{
Ansiedade de provas em estudantes do Ensino Médio
}

\author{
Test anxiety on High School students
}

Luiz Ricardo Vieira Gonzaga [a], Andressa Melina Becker da Silva [b], Sônia Regina Fiorim Enumo[c],

[a] Doutor em Psicologia pelo pela Pontifícia Universidade Católica de Campinas, Campinas, SP- Brasil, e-mail:

tccricardogonzaga@yahoo.com.br

[b]Doutora em Psicologia pela Pontifícia Universidade Católica de Campinas, Campinas, SP- Brasil, Professora de

Graduação em Psicologia da Universidade de Sorocaba, Sorocaba, SP-Brasil, e-mail: andres-

sa_becker@homtail.com

[c] Doutora em Psicologia, Professora do Programa de Pós-Graduação em Psicologia e do Programa de Pós-

Graduação em Ciências da Saúde da Pontifícia Universidade Católica de Campinas, Campinas, SP-Brasil, e-mail: sonia.enumo@puc-campinas.edu.br

\section{Resumo}

O problema da ansiedade de provas tem despertado interesse no meio educacional por haver uma prevalência de $30 \%$ a $33 \%$ dos estudantes com algum tipo de ansiedade severa, uma condição caracterizada no contexto acadêmico como "ansiedade de provas" (testanxiety). Esta inclui reações psicofisiológicas e comportamentais associadas ao medo do fracasso em situações de avaliação. Estudantes com alta ansiedade de provas têm mais dificuldade de aprendizagem, de retenção de novos conteúdos e mau desempenho acadêmico, estando mais propensos à evasão escolar. Este estudo avaliou o nível de ansiedade de provas em estudantes do Ensino Médio (EM) de uma escola pública de São Paulo. Participaram 379 estudantes do $1^{\circ}$ ao $3^{\circ}$ ano do EM, com idade entre $14-20$ anos $(\mathrm{M}=16,27 ; \pm 1,04)$, que responderam o 
Test AnxietyScale (TAS) adaptado e validado para esta pesquisa, contendo 37 itens. Procedeu-se uma análise descritiva e correlacional (Teste Qui-Quadrado). Os resultados mostraram uma prevalência de ansiedade de provas em $62,53 \%$ da amostra, com diferença significativa em favor das meninas $(66,96 \%)$, quando comparadas aos meninos (55,92\%). Os alunos do $2^{\circ}$ Ano, seguidos do $3^{\circ}$ Ano e os mais velhos apresentaram mais ansiedade de prova. Os estressores relacionados às provas referiram-se à autocobrança de desempenho, sentimento de incapacidade e reações psicofisiológicas. Os resultados sinalizam a importância de identificar e analisar a inter-relação das variáveis da pessoa, como as estratégias de enfrentamento e o manejo de situações estressoras, e a avaliação escolar, dados os reflexos no desempenho acadêmico.

Palavras-chave: Ansiedade. Avaliação Educacional. Ensino Fundamental e Médio. Estudantes.

\begin{abstract}
The issue of test anxiety has raised interest on educational environment because of a $30 \%$ to $33 \%$ prevalence of students with some type of severe anxiety, a condition characterized by the academic community as "test anxiety". This includes psicological, physical and behavioral reactions associated with fear of failure in assessment situations. Students with high test anxiety report having more learning issues, difficulty retaining new contents and poor academic performance, wich makes them more likely to evade school. This research assessed test anxiety levels on High School students (EM) from a public school at São Paulo. 379 students ranging from EM's 1st to 3rdyears, whose age range from 14 to 20 years old $(A=16.27 ; \pm 1.04)$ who answered the adapted and validated Test Anxiety Scale (TAS) instrument used for this research, which contains 37 items. A descriptive and correlational analysis was performed (ChiSquare test). Results demonstrated that test anxiety prevails on $62.53 \%$ of subjects, showing significant difference towards girls (66.96\%) when compared to boys (55.92\%). 2nd year students, followed by $3 \mathrm{rd}$ years, and older students presented higher test anxiety. Stressful sources related to performance demands made by themselves, feelings of inaptitude and physical and psycological reactions. Results point to the importance of identifying and analysing the interrelation between a person's variables such as its coping mechanisms and management when facing stressful situations and school assessments given they reflect on academic performance.
\end{abstract}

Keywords: Anxiety.Educational Measurement.Education Primary and Secondary. Students

\title{
Introdução
}

O problema da ansiedade de provas tem despertado interesse no meio educacional por haver uma prevalência de $30 \%$ a $33 \%$ dos estudantes com algum tipo de ansiedade severa, uma condição caracterizada no contexto acadêmico como "ansiedade de provas" (testanxiety) (Methia, 2004; Sarason, 1978; Spielberger\&Vagg, 1995; Zeidner, 1998). Muitas decisões importantes acabam relacionadas ao desempenho em provas escolares, acadêmicas e seletivas no campo da escola, universidade e trabalho, por exemplo. A prova é amplamente utilizada nos campos da educação, organização, governo e setores militares para auxiliar na tomada de decisão sobre as pessoas em relação à sua realização, competências, carreira acadêmica e habilidades (Herzer, Wendt, \& Hamm, 2015; Rana \& Mahmood, 2010; Zeidner, 1998). Evidencia-se a "cultura da prova", na qual o futuro do indivíduo é, em parte, influenciado e determinado pelo seu desempenho e rendimento no mesmo (Embse \& Hasson, 2012). Dessa maneira, quando se consideram os vários usos das provas em nossa cultura, e as maneiras pelas quais elas podem determinar a vida das pessoas, não é surpresa que a situação de prova possa evocar reações de ansiedade em muitos indivíduos de todas as idades. Precocemente, muitas crianças tornam-se orientadas para a prova e ansiosas por causa dela (Zeidner, 1998). Dados apontam que a prevalência da ansiedade em prova entre as crianças em idade escolar pode variar de $10 \%$ até $30 \%$ (King \& Ollendick, 1989). 
Ansiedade de provas compreende reações psicológicas, fisiológicas e comportamentais que ocorrem em associação com a preocupação com os resultados negativos resultantes do fracasso ou mau desempenho em situações de avaliação antes (fase antecipatória), durante (fase de confrontação) e depois de um período de exames (fase de espera) (Zeidner, 1998). A fase antecipatória constitui-se de um período caracterizado pela iminência da situação avaliativa. De fato, com a aproximação de uma prova, o indivíduo está preocupado com as demandas, possibilidades e constrangimentos relacionados a ela e pensa em como se preparar e regular os sentimentos e emoções associadas com a avaliação negativa. A incerteza dos resultados é alta, nesta fase, podendo ser experimentada emoções ameaçadoras e/ou desafiadoras. A fase de confrontação se desenvolve durante o enfrentamento com o estressor (por exemplo: em uma situação de prova). A ansiedade, particularmente como seu componente emocional, atinge um pico durante os primeiros momentos de contato com o exame, enquanto a preocupação, o componente cognitivo da ansiedade frente aos exames, torna-se mais estável durante o processo de avaliação. A fase de espera refere-se ao período do pós-teste, quando os resultados ainda não foram divulgados. A incerteza em relação à natureza dos exames já foi resolvida ou reduzida significativamente, embora os indivíduos ainda possam experimentar apreensão com os resultados acadêmicos. Por fim, na fase dos resultados, uma vez que são divulgadas as notas, a incerteza é resolvida e as preocupações dos estudantes são direcionadas para o significado e a importância dos resultados obtidos (Folkman \& Lazarus, 1984).

A ansiedade em provas refere-se também à tendência do indivíduo para analisar uma situação de avaliação de desempenho como uma ameaça pessoal (Spielberger \& Vagg, 1995). Como resultado, o indivíduo tende a reagir com percepções de ameaças, sentimentos reduzidos de autoeficácia, cognições autodepreciativas, atribuições de falha por antecipação, reações emocionais mais intensas e excitação logo no primeiro sinal de fracasso (Sarason \& Sarason, 1990). Neste enfoque, ansiedade de provas tem sido compreendida como sinônimo do medo do fracasso em situações de avaliação (Sarason, 1978; Zeidner, 1998). Já o comportamento de ansiedade em provas é geralmente evocado quando uma pessoa acredita que ela ou as suas capacidades intelectuais, motivacionais e sociais são ultrapassadas por demandas decorrentes da situação de avaliação (Sarason \& Sarason, 1990). Este ponto de vista sustenta que os alunos com ansiedade de provas podem ter um mau desempenho devido à incapacidade para interpretar, organizar, ou compreender de forma eficaz o conteúdo a ser usado durante a situação avaliativa (Cassady \& Finch, 2015).

Uma forma alternativa de conceituar os diferentes componentes da ansiedade de provas é apresentada em um modelo biopsicossocial, que destaca três processos diferentes envolvidos, incluindo: o comportamento, a cognição (pensamentos) e os aspectos fisiológicos do indivíduo (Loweet al., 2008). O comportamento refere-se à forma como o indivíduo age em situações de avaliação, incluindo tanto tarefas relevantes (por exemplo, focar a atenção na tarefa), quanto a comportamentos irrelevantes (por exemplo, folhear itens, procrastinação e esquiva da atividade). As reações fisiológicas correspondem à excitação autonômica que ocorre durante a situação de avaliação, que incluem a excitação emocional (por exemplo, aumento do ritmo cardíaco ou tensão muscular). A cognição inclui pensamentos negativos que interferem na tarefa (por exemplo, pensamentos sobre a humilhação social, autodepreciativos ou consequências do fracasso) (Loweet al., 2008; Zeidner, 1998). Esses processos inter-relacionados contribuem para o desenvolvimento e a expressão de 
ansiedade em provas (Loweet al., 2008). Além disso, quando um estudante com ansiedade em provas avalia um teste como uma ameaça, ele o executa de uma forma menos otimizada, as cognições inadequadas do aluno são reforçadas e a ansiedade a prova aumenta. Esta avaliação surge, por um lado, do processo de negociação entre as demandas e recursos do meio ambiente e, por outro, das crenças, prioridades e objetivos do aluno, resultando em uma reação emocional, cuja intensidade depende da percepção de ameaça à segurança, autoestima e estabilidade pessoal. Esta resposta à ansiedade de prova pode ser provocada por ambas as situações ou estímulos externos, e pelos estímulos internos, como pensamentos, crenças, expectativas, responsabilidades, entre outros, que são percebidos pelo sujeito como eventos ameaçadores (Loweet al., 2008; Methia, 2004). Segundo Serrano-Pintado e Escolar-Llamazares (2014), a identificação de diferentes perfis de ansiedade de provas e das suas necessidades são essenciais para se desenvolver estratégias de tratamento eficazes.

Nota-se que a ansiedade de prova é comum em jovens em idade escolar, especialmente do sexo feminino (Loweet al., 2008; Liu, 2012), esta constantemente relacionada com dificuldades acadêmicas associada também a problemas internalizantes como sintomas de Transtornos de Ansiedade, Transtorno Depressivo e Transtorno de Pânico (Weems et al., 2014). É considerada como uma forma de ansiedade subclínica, compartilhando características similares com outros tipos de ansiedade de avaliações sociais, incluindo ansiedade competitiva em esportes (relacionada a atletas), ansiedade de atuação (relacionado a artistas), ansiedade de computador, matemática, estatística e de falar em público, por exemplo (Zeidner\& Mathews, 2005). De acordo com o Manual de Diagnóstico e Estatístico dos Transtornos Mentais- $5^{\mathrm{a}}$ edição (DSM- 5, 2013), tais temores intensos relacionados ao desempenho manifestados em ambientes acadêmicos pode ser diagnosticado como um transtorno de ansiedade social, com o componente especificador de desempenho apenas se todos os critérios clínicos forem satisfeitos. Contudo, podem ser indicadores de diversas psicopatologias, como transtornos de ansiedade e depressão, podendo ocorrer casos de suicídio (Latifaet al., 2012; Zeidner, 1998).

Algumas pesquisas referentes a esse constructo apontam a importância no estudo de alguns processos associados, principalmente na sua relação com o desempenho acadêmico (Chapellet al., 2005). A pesquisa realizada por Rana e Mahmood (2010) observou a relação entre a ansiedade de teste e o desempenho acadêmico entre 400 adolescentes no Irã, na faixa etária dos 15 aos 19 anos de idade. Algumas escalas foram aplicadas como o Test Anxiety Inventory [TAI] e o Grade Point Average [GPA] e apontaram resultados significativos entre a ansiedade de teste e o desempenho acadêmico entre os adolescentes. Houve uma correlação negativa, de forma que, quando a ansiedade de provas aumentava, o desempenho acadêmico dos alunos diminuía, proporcionalmente. Esse resultado foi corroborado com os estudos de Chapellet al. (2005) que descobriram que os estudantes com baixa ansiedade de prova tiveram maior desempenho acadêmico do que os alunos com um nível moderado e superior em ansiedade de provas. Uma das razões pelas quais a ansiedade de prova tem impacto no desempenho acadêmico é a sua influência na atenção e concentração, os quais, mais tarde, têm efeito sobre a memória e o desempenho acadêmico.

Em relação à influência da ansiedade de prova na memória dos estudantes, um estudo feito por Yousefi e Redzuan (2010), com a aplicação do TAI e do WechslerMemoryScale [WMS-III] em 400 estudantes do Ensino Médio, apontou uma correlação significativa entre a ansiedade de prova e a memória. Segundo os mesmos autores, a conclusão 
desse estudo implica que a ansiedade de prova pode afetar a memória dos adolescentes. Além disso, a ansiedade de prova pode influenciar na motivação para o aprendizado e na capacidade de prestar atenção e na concentração podendo resultar no mau desempenho acadêmico. Outras pesquisas convergem para esta mesma temática e evidenciam as relações entre a ansiedade de prova a outros fatores multivariados, como as relações entre o mau gerenciamento do tempo e a ansiedade em provas (Latifa, Nermim, \& Sanaa, 2012); entre os estressores autorrelatados (teste de ansiedade, por exemplo) e índices elevados de pressão arterial (Conley\& Lehman, 2012); a transição do Ensino Fundamental de adolescentes para o Ensino Médio e os resultados acadêmicos (desempenho acadêmico, desengajamento acadêmico, motivação acadêmica e ansiedade de provas) (Goldstein, Boxer, \& Rudolph, 2015); as diferenças culturais e de gênero na expressão da ansiedade de provas em diversos países (Nyrooset al., 2015); a influência do medo promovido pelos professores e de apelos de eficácia em estudantes universitários em situações avaliativas (Embse, Schultz, \&Draughn, 2015); as diferenças no desenvolvimento do papel dos pensamentos automáticos e da ansiedade de provas teriam no desempenho acadêmico baseado no modelo Cognitivo-Comportamental (Miloseva, 2012); a identificação de subcomponentes cognitivos referentes ao constructo ansiedade de provas em estudantes (Cassady\&Finch, 2015); as diferenças na forma das pessoas lidarem com o estressor ansiedade de provas (Strack\& Esteves, 2015); as diferenças atribuídas entre a ansiedade de avaliação na modalidade escrita e oral em estudantes do Ensino Médio (Sparfeld, Rost, Baumeister, \&Christ, 2013); as relações entre ansiedade de provas, autoeficácia, processos autoregulatórios e desempenho acadêmico em estudantes (Schnell et al., 2015); entre a situação de avaliação, ansiedade, coping (enfrentamento) e procrastinação durante um período de provas (Lay, Edwards, Parker, \&Endler, 1989) e, por fim, nas relações entre o estresse percebido, neuroticismo e a ansiedade de teste (Hoferichter, Raufelder, \& Eid, 2014).

Apesar do constructo ser estudado desde 1952, com os trabalhos de Sarason e Mandler, no contexto brasileiro, a literatura é incipiente nesta área, mesmo com o crescente interesse que esse constructo exerce quando se abordam variáveis como o rendimento acadêmico e a motivação escolar (Bzuneck\& Silva, 1989).

Nesse contexto acadêmico de ansiedade de avaliação acadêmica, objetivou-se neste estudo analisar o nível de ansiedade de provas escolares em estudantes do Ensino Médio de uma escola pública da capital do Estado de São Paulo.

\section{Método}

Para alcançar os objetivos propostos da pesquisa foi realizado um estudo do tipo descritivo e correlacional.

\section{Participantes}

Participaram deste estudo 379 adolescentes, 227 meninas (59,89\%) e 152 meninos (40,10\%), com idade entre 14 e 20 anos $(M=16,00 ; \pm 1,03)$, sendo que $115(30,34 \%)$ cursavam o $1^{\circ}$ ano, $144(37,99 \%)$ o $2^{\circ}$ Ano e 120 (31,66\%) o $3^{\circ}$ Ano do Ensino Médio [EM]. A coleta de dados foi realizada em uma escola pública de Ensino Médio, localizada 
no bairro da Lapa, capital do Estado de São Paulo, com a autorização da Direção da escola. Esta escola abrange o Ensino Fundamental ( $6^{\circ}$ ao $9^{\circ}$ Ano), Médio ( $1^{\circ}, 2^{\circ}$ e $3^{\circ}$ Ano) e o EJA (Ensino para Jovens e Adultos) do $1^{\circ}$ ao $3^{\circ}$ Termo e é mantida pela Secretaria de Estado da Educação de São Paulo. É composta por 17 salas de aula com capacidade de receber mais de 2.040 alunos nos três períodos de atuação. O quadro discente é composto por 567 alunos, sendo que 190 cursam o $1^{\circ}$ Ano, divididos em quatro salas de aula; 209 no $2^{\circ}$ Ano, divididos em quatro salas de aula; e 168 no $3^{\circ}$ Ano, divididos em três salas de aula. Dispõe também de duas quadras poliesportivas com vestiário, laboratório de ciências, biblioteca, sala de informática, auditório e páteo coberto 2.2. Instrumentos e materiais

Para medir a ansiedade em provas escolares, foi adaptado e aplicado o Test AnxietyScale [TAS] (Sarason, 1978). O TAS contém 37 itens para o estudante assinalar Verdadeiro ou Falso, com itens como: “ a) Quando estou fazendo uma prova importante, fico pensando no quanto os outros estudantes são melhores do que eu; b) Quando estou fazendo uma prova importante, costumo transpirar bastante e; c) Eu prefico fazer um trabalho a uma prova para conseguir nota em uma disciplina”. A escala avalia a ocorrência do evento nos últimos seis meses e o nível de agradabilidade (desejabilidade) deste. Esta escala esta embasada em uma perspectiva cognitiva, que define a ansiedade como um tipo de resposta cognitiva marcada por autodúvida, sentimentos de inadequação e autoculpa (Sarason, 1978). Ela avalia aspectos cognitivos, fisiológicos e comportamentais. A versão original do instrumento apresenta sensibilidade e confiabilidade $(\alpha=0,87)$, com intervalos de teste-reteste de várias semanas. A pontuação do TAS é calculada através da média estipulada durante a validação do instrumento, que seria de $M=16,72$ para homens e $M=19,74$ para mulheres. Assim, escores acima da média são indicativos de ansiedade de provas e abaixo destes, sem ansiedade de provas. Os resultados foram analisados como variáveis numérico-discretas, utilizando a soma das notas de cada item.

Antes da aplicação do instrumento, foi feita uma aplicação-piloto com 15 adolescentes (alunos do $3^{\circ}$ Ano do EM), que não foram participantes da pesquisa, com o objetivo de observar a clareza, compreensão e a adaptação do questionário ao ambiente acadêmico e o aspecto cultural em português. Nesta fase, foi investigado se houve alguma dificuldade durante o preenchimento e as dificuldades na compreensão de frases e palavras. Foi ressaltado o cuidado por parte do aplicador, na garantia de que os respondentes compreendessem de forma similar as perguntas do questionário. Por fim, foi realizada uma revisão geral para garantir que não houvesse quaisquer erros de pontuação ou concordância, de forma que o questionário traduzido respeitasse a versão do original.

Após a validação cultural e lingüísticado TAS, este foi aplicado em 378 estudantes e o instrumento apresentou uma alta consistência interna para o total dos itens $(\alpha=0,85)$.

\section{Procedimento}

Os procedimentos realizados durante o presente estudo respeitaram a legislação sobre a pesquisa com seres humanos (Resolução 466/2012, do Conselho Nacional de Saúde) e as normas do Conselho Federal de Psicologia (Dezembro, 2000), com a aprovação do Comitê de Ética da universidade (Parecer n. 1.055.076) e a autorização da coordenação escolar, mediante a anuência dos responsáveis e/ou participantes, pela assinatura do Termo de Consentimento Livre e Esclarecido. Em um primeiro momento, foi enviada uma carta 
de anuência à Direção Escolar explicando os objetivos do estudo. Após a permissão, os estudantes do Ensino Médio ( $1^{\circ}, 2^{\circ}$ e $3^{\circ}$ Ano) foram convidados a participarem de forma voluntária, recebendo ao final do processo de coleta de dados uma cartilha devolutiva com informações e estratégias de prevenção à ansiedade de provas acadêmicas. Assim, os 379 participantes interessados assinaram o Termo de Consentimento Livre e Esclarecido (TCLE) e foi aplicado o questionário. Observou-se que, quando estes eram menores de idade, o TCLE era assinado pelos responsáveis. A coleta de dados foi realizada por classe, de forma coletiva, com umencontro, com a duração aproximada de $60 \mathrm{~min}$.

Para descrever o perfil da amostra para as variáveis em estudo, foram feitas tabelas de frequência das variáveis categóricas (sexo, idade e escolaridade), com os valores de frequência absoluta (n) e percentual (\%), e as estatísticas descritivas das variáveis numéricas (idade, resultado da escala, por ex.), com os valores de média e o desvio padrão. Para analisar a consistência interna das escalas foi utilizado o coeficiente alfa de Cronbach. Valores de alfa maiores que 0,70 indicam alta consistência e confiabilidade da escala. Para comparar as variáveis categóricas entre os alunos com e sem ansiedade de provas foi utilizado o teste Qui-Quadrado. O nível de significância adotado para os testes estatísticos foi de 5\%.Para a realização das análises estatísticas foi utilizado o software StatisticalAnalysis System [SAS] para Windows@, versão 9.2 para facilitar as análises e correlações necessárias para embasar a pesquisa.

\section{Resultados}

$\mathrm{Na}$ amostra avaliada pelo TAS $(\mathrm{N}=379)$, houve uma prevalência de $62,53 \%(\mathrm{n}=$ 237) de alunos com ansiedade de provas, com maior prevalência de alunas, com diferença significativa pelo teste Qui-Quadrado. Em comparação com as séries e ansiedade de provas, a prevalência foi maior no $1^{\circ}$ Ano, seguido do $3^{\circ}$ Ano, embora sem diferença significativa. Houve uma maior frequência de alunos mais novos com ansiedade de provas, embora também sem diferença significativa pelo teste Qui-Quadrado (Tabela 1).

Observou-se que a média de ansiedade de provas do sexo feminino foi maior que a do sexo masculino, tanto para a amostra com ansiedade quanto para sem ansiedade. Já em relação à idade, a prevalência da média foi maior para os alunos com ansiedade, acima de 17 anos, enquanto para os sem ansiedade, a maior média foi para os alunos com idade entre 14 e 15 anos. Quanto às séries escolares, a maior média para alunos com ansiedade foi para o Terceiro Ano, enquanto que a maior média para os sem ansiedade foi no Primeiro Ano (Tabela 1) 
Tabela 1. Frequência dos alunos do Ensino Médio com ansiedade de provas, por gênero, idade e série $(\mathrm{N}=379)$

\begin{tabular}{|c|c|c|c|c|c|}
\hline Variáveis & $\begin{array}{l}\text { Com ansie- } \\
\text { dade de } \\
\text { provas } \\
n(\%)\end{array}$ & $\begin{array}{l}\text { Sem ansie- } \\
\text { dade de } \\
\text { provas }\end{array}$ & $\begin{array}{l}\text { Média com } \\
\text { ansiedade de } \\
\text { provas (DP) }\end{array}$ & $\begin{array}{l}\text { Média sem ansi- } \\
\text { edade de provas } \\
\text { (DP) }\end{array}$ & $\begin{array}{l}* p- \\
\text { valor }\end{array}$ \\
\hline \multicolumn{6}{|l|}{ Gênero } \\
\hline $\begin{array}{l}\text { Feminino } \\
(\mathrm{n}=227)\end{array}$ & $152(66,96)$ & $75(33,04)$ & $25,91( \pm 3,67)$ & $16,00( \pm 3,22)$ & \multirow[t]{3}{*}{$0,030 *$} \\
\hline $\begin{array}{l}\text { Masculino } \\
(\mathrm{n}=152)\end{array}$ & $85(55,92)$ & $67(44,08)$ & $21,74( \pm 4,32)$ & $11,00( \pm 3,36)$ & \\
\hline Total & $237(62,53)$ & & & & \\
\hline \multicolumn{6}{|l|}{ Idade } \\
\hline $\begin{array}{l}14-15 \text { anos } \\
(\mathrm{n}=88)\end{array}$ & $62(70,45)$ & $26(29,55)$ & $23,10( \pm 3,67)$ & $15,00( \pm 4,14)$ & \multirow[t]{4}{*}{0,209} \\
\hline $\begin{array}{c}16 \text { anos } \\
(n=138)\end{array}$ & $82(59,42)$ & $56(40,58)$ & $24,82( \pm 4,56)$ & $13,00( \pm 3,90)$ & \\
\hline $\begin{array}{l}\geq 17 \\
(n=153)\end{array}$ & $93(60,78)$ & $60(39,22)$ & $24,84( \pm 4,58)$ & $13,00( \pm 3,94)$ & \\
\hline Total & $237(62,53)$ & $142(37,47)$ & & & \\
\hline \multicolumn{6}{|l|}{ Série } \\
\hline $\begin{array}{l}1^{\circ} \text { Ano } \\
(n=115)\end{array}$ & $75(65,22)$ & $40(34,78)$ & $23,28( \pm 4,06)$ & $14,50( \pm 416)$ & \multirow[t]{4}{*}{0,647} \\
\hline $\begin{array}{l}2^{\circ} \text { Ano } \\
(n=144)\end{array}$ & $86(59,72)$ & $58(40,28)$ & $24,58( \pm 4,59)$ & $13,00( \pm 3,91)$ & \\
\hline $\begin{array}{l}3^{\circ} \text { Ano } \\
(n=120)\end{array}$ & $76(63,33)$ & $44(36,67)$ & $25,22( \pm 4,34)$ & $13,00( \pm 3,85)$ & \\
\hline $\begin{array}{l}\text { Total } \\
(\mathrm{N}=379)\end{array}$ & $237(62,53)$ & $142(37,47)$ & $24,38( \pm 4,40)$ & $13,31( \pm 3,94)$ & \\
\hline
\end{tabular}

Feita à análise comparativa entre o gênero e as séries, as alunas do $3^{\circ}$ Ano e as do $1^{\circ}$ Ano apresentaram mais ansiedade de provas. Houve diferença significativa das alunas do $3^{\circ}$ Ano pelo teste Qui-Quadrado (Tabela 2).

Tabela 2. Frequência dos alunos do Ensino Médio com ansiedade, por gênero e série escolar $(N=379)$

\begin{tabular}{lllll}
\hline Série & Feminino & $\begin{array}{l}\text { Masculino } \\
n(\%)\end{array}$ & Total & $* \boldsymbol{p}$-valor \\
\hline $1^{\circ}$ Ano & $35(70,00)$ & $40(61,54)$ & 75 & 0,345 \\
$2^{\circ}$ Ano & $60(62,50)$ & $26(54,17)$ & 86 & 0,337 \\
$3^{\circ}$ Ano & $57(70,37)$ & $19(48,72)$ & 76 & $0,021^{*}$ \\
\hline Total & 152 & 85 & 237 & \\
\hline
\end{tabular}

Nota: ${ }^{*} p \leq 0,05=*$ Valores significativos pelo Teste Qui-Quadrado. 
Considerando o que o aluno avaliou como estressores internos e externos de ansiedade de provas em relação às situações percebidas como "ruins" por eles, com valores de frequência absoluta (n) e percentual (\%), observou-se um percentual de ocorrência variando de $16,89 \%$ a $89,79 \%$ em relação aos estressores acadêmicos. Optou-se pela categorização das respostas dos itens. Assim, alguns estressores acadêmicos apontados com maior frequência pelos alunos estavam relacionados a:

Autocobrança no desempenho da prova: "querer fazer o melhor na prova" (89,79\%), "ficar imaginando se passará na matéria" (75,79\%), "ter um melhor desempenho sem a cobrança do tempo" (73,42\%);

Sentimento de incapacidade: "preferência em fazer um trabalho ao invés de uma prova" (81,58\%), "preocupação em participar de um teste de inteligência" $(73,82 \%)$, "crença de fracasso diante da prova" (73,82\%);

Reações psicofisiológicas perante a situação de avaliação acadêmica: "nervosismo diante da prova influenciando no seu desempenho acadêmico" $(83,11 \%)$, "sentir-se tenso diante da prova" (81\%), "preferir que as provas não incomodassem tanto" $(73,42 \%)$.

Por outro lado, foi observada também a prevalência de um indicador positivo frente à avaliação acadêmica, que envolveu a "autoconfiança e o relaxamento diante da situação de avaliação" $(73,56 \%)$.

\section{Discussão}

A presente pesquisa objetivou analisar o nível de ansiedade de provas escolares em estudantes do Ensino Médio ( $1^{\circ}, 2^{\circ}$ e $3^{\circ}$ Ano) em uma escola estadual do Estado de São Paulo.

Observou-se que, mais da metade da amostra de 379 alunos apresentaram ansiedade de provas com destaque para as meninas, especialmente as alunas do $3^{\circ}$ Ano, sendo seguidas pelas alunas do $1^{\circ}$ Ano. No que se refere aos dados apontados com relação às séries escolares, a literatura internacional aponta que mais de 33\% dos alunos do Ensino Fundamental e Médio experimentam alguma ansiedade de prova (Methia, 2004). Quanto à diferença de gênero na ansiedade de provas esse dado vai ao encontro aos da literatura (Chapellet al., 2005; Liu, 2012; Lowe et al., 2008). Esta condição pode estar relacionada a uma tendência geral das mulheres perceberem as situações avaliativas de provas como uma ameaça ao self ao invés de interpretarem como um desafio, aumentando, desta maneira, sua crença de incapacidade diante da prova (Lay, Edwards, Parker, \&Endler, 1989). Ao lidar com a situação acadêmica como uma ameaça, o aluno poderá desencadear estratégias mal adaptativas que poderão influenciar no modo deles lidarem com a situação avaliativa, desencadeando, consequentemente, sintomas psicofisiológicos diante da situação de avaliação. 
Quanto aos estressores acadêmicos apontados com maior freqüência pelos estudantes houve uma prevalência de estressores internos: autocobrança no desempenho da prova (querer fazer o melhor na prova, por exemplo) e sentimento de incapacidade (preferência em fazer um trabalho ao invés de uma prova, por exemplo) bem como reações psicofisiológicas (sentir-se tenso diante da prova, por exemplo). Observa-se em alguns estudos que vários fatores biopsicossociais, incluindo o fator biológico (por exemplo, excitação fisiológica), psicológico (fatores emocionais e cognitivos) e social (por exemplo, a pressão dos pais), contribuem para o desenvolvimento e a expressão de ansiedade em provas (Loweet al., 2008). Fatores internos e externos como estudar para as provas, a própria situação de avaliação, a cobrança por um bom desempenho acadêmico, o excesso de atividades escolares a serem feitas e a necessidade de sair-se bem na prova, por exemplo, foram apontadas por serem as mais importantes fontes de estresse para os alunos do Ensino Médio (Loweet al., 2008; Methia, 2004).

Apesar do tamanho amostral deste estudo, deve-se considerar a necessidade de mais estudos, incluindo escolas privadas. Cabe, porém, lembrar que a maioria da nossa população usa o ensino público. Em face das limitações apresentadas, seria importante e necessária a realização de estudos controlados, com amostra randomizadas e não de conveniência como esta, apesar do grande número de participantes.

\section{Conclusões}

Observou-se nos resultados deste estudo que as situações avaliativas, como provas escolares, podem ser estressores e desencadear algum nível de ansiedade nos estudantes, com possíveis consequências no rendimento acadêmico. Essa condição mostra a importância de intervenções nessa fonte de estresse em estudantes do Ensino Médio. As intervenções podem ter um caráter psicoeducativo, ensinando os alunos a lidar de forma funcional com o contexto de provas escolares. Aprender a utilizar estratégias adaptativas poderá ser um modo de se prevenir diante de outras situações identificadas como estressoras por esses estudantes. Podem também ser preventivas de quadros psicopatológicos, como os transtornos de ansiedade e depressão. Nessas intervenções, é importante considerar as diferenças de gênero e idade, pois, neste estudo, a ansiedade de provas teve uma prevalência nas mulheres, em alunos do $1^{\circ}$ e $3^{\circ}$ Ano e mais novos.

Estes dados sinalizaram a importância de analisara inter-relação entre as variáveis da pessoa, como as estratégias de enfrentamento e o manejo de situações estressoras, e a avaliação acadêmica. Estudos sobre o tema podem auxiliar na identificação dos determinantes das estratégias de enfrentamento utilizadas pelos alunos em situações avaliativas, com reflexos no desempenho acadêmico. Assim, problemas de desenvolvimento e de comportamento poderiam ser melhor detectados e prevenidos. 
Nota. Trabalho derivado da tese de doutorado do primeiro autor, orientada pelo último: "Enfrentando Provas Escolares: Relações com Problemas de Comportamento e Rendimento Acadêmico no Ensino Médio"

Agradecimentos -Aos responsáveis, funcionários e alunos da instituição escolar;ao estatístico Helymar C. Machado; às agências de fomento à pesquisa - CAPES (bolsa de doutorado para os dois primeiros autores) e CNPq (bolsa de produtividade em pesquisa em nível 1B para a última autora; auxílio à pesquisa - Proc. no 455543-2014-3).

\section{Referências}

American Psychiatric Association. (2013). Diagnostic and Statistical Manual of Mental Disorders: DSM-5 (5th ed.).

Bzuneck, J.A., \& Silva, R. (1989). 0 problema da ansiedade nas provas: Perspectivas contemporâneas, Semina, 10(3), 190-195.

Cassady, J.C., \& Finch, W.H.(2015). Using factor mixture modeling to identify dimensions of cognitive test anxiety.Learning and Individual Differences, 41, 14-20. doi: http://dx.doi.org/10.1016/j.lindif.2015.06.002.

Chapell, M. S., Blanding, Z. B., Takahashi, M., Silverstein, M. E., Newman, B., Gubi, A., \&Mccann, N. (2005). Test anxiety and academic performance in undergraduate and graduate students. Journal of Educational Psychology, 97(2), 268-274. doi: http://10.1037/0022.0663.97.2.268

Conley, K. M., \& Lehman, B. J. (2012).Test anxiety and cardiovascular responses to daily academic stressors.Stress and Health, 28(1), 41-50. doi: http://10.1002/smi.1399.

Embse, N.P.V.D., \&Hasson, R.. (2012). Test anxiety and high-stakes test performance between school settings: Implications for educators. PreventingSchoolFailure, 56(3), 180-187. doi: 10.1080/1045988X.2011.633285.

Embse, N.P.V.D., Schultz, B.K., Draughn, J.D.(2015). Readying students to test: The influence of fear and efficacy appeals on anxiety and test performance.SchoolPsychologyInternational, 36(6), 620-637. doi: 10.1177/0143034315609094

Goldstein, S.E., Boxer, P., \& Rudolph, E. (2015). Middle school transition stress: Links with academic performance, motivation, and school experiences, Contemporary School Psychology, 19, 21-29. doi: 10.1007/s40688-014-0044-4

Herzer, F.; Wendt, J., \& Hamm, A.O. (2015). Discriminant validity of constructs derived from the self-regulative model for evaluation anxiety for predicting clinical manifesta- 
tions of test anxiety. Behaviour Research and Therapy, 73, 52-57. doi: http://dx.doi.org/10.1016/j.brat.2015.07.012

Hoferichter, F., Raufelder, D., \&Eid, M. (2014).The mediating role of socio-motivacional relationships in the interplay of perceived stress, neuroticism, and test anxiety among adolescent students.Psychology in the Schools, 51(7), doi: 10.1002/pits.21778.

King, N. J., \&Ollendick, T. H. (1989). Children's anxiety and phobic disorders in school settings: Classification, assessment, and intervention issues. Review of Educational Research,59(4), 431 - 470. doi: http://10.3102/00346543059004431.

Latifa, T. A. A., Nermim, M. E., \&Sanaa, M. S. (2012) Test anxiety, and skills of time management among faculty nursing students.Journal of American Science, 8(4), 261-269.

Lazarus, R., \&Folkman, S. (1984). Stress, appraisal, and coping. New York: Springer.Liu, Y.Y. (2012). Students' perceptions of school climate and trait test anxiety. Psychological Reports: Sociocultural Issues in Psychology, 111(3), 761-764.

Lowe, P. A., Lee, S. W., Witteborg, K. M., Prichard, K. W., Luhr, M. E., Cullinan, C. M., \&Janik, M. (2008). The Test Anxiety Inventory for Children and Adolescents (TAICA): Examination of the psychometric properties of a new multidimensional measure of test anxiety among elementary and secondary school students. Journal of Psychoeducational Assessment, 26(3), 215-230. doi: http://10.1177/0734282907303760.

Methia, D. (2004). Help your child overcome test anxiety and achieve higher test scores. College Station: Virtualbook.worm.

Miloseva, L. (2012). Can automatic thoughts and test anxiety explain school success and satisfaction in adolescents? PrimenjenaPsihologija, 1, 43-57.

Nyroos, M., Korhonen, J., Peng, A., Linnanmäki, K., Svens- Liavag, C., Bagger, A., \& Gunnar, S. (2015). Cultural and gender differences in experiences and expression of test anxiety among Chinese, Finnish, and Swedish grade 3 pupils. International Journal of School \& Educational Psychology, 3(1), 37-48. doi: 10.1080/21683603.2014.915773

Rana, R., \&Mahmood, N. (2010).The relationship between test anxiety and academic achievement.BulletinofEducationandResearch, 32(2), 63-74.

Sarason, I. G. (1978). The test anxiety scale: Concept and research. In C. D Spielberger, \& I. G. Sarason (Eds.), Stress and anxiety (v. 5, pp.193-216). Washington: Hemisphere.

Sarason, I. G., \&Sarason, B. R. (1990). Test anxiety. In H. Leitenberg (Ed.), Handbook of social and evaluative anxiety (pp.475-496). New York: Plenum Press.

Schnell, K., Ringeisen, T., Raufelder, D., Rohrmann, S. (2015). The impact of adolescents' self-efficacy and self-regulated goal attainment processes on school performance Do gender and test anxiety matter?,Learning and Individual Differences, 38, 90-98. doi: http://dx.doi.org/10.1016/j.lindif.2014.12.008 
Serrano-Pintado, I.,\& Escolar-Llamazares, M.C. (2014). Description of the general procedure of a stress inoculation program to cope with the test anxiety.Psychology, 5, 956965. doi: http://dx.doi.org/10.4236/psych.2014.58106.

Sparfeldt, J.R., Rost, D.H., Baumeister, U.M., Christ, O. (2013). Test anxiety in written and oral examinations. Learning and Individual Differences, 24, 198-203.

Spielberger, C. D., \&Vagg, P. R. (1995). Test anxiety: A transactional process. In C. D. Spielberger, \& P. R. Vagg (Eds.), Test anxiety: Theory, assessment, and treatment (pp. 3-14).

Whashington: Taylor \&Francis.Strack, J., \&Esteves, F. (2015). Exams? Why worry? Interpreting anxiety as facilitative and stress appraisals, Anxiety, Stress, \& Coping: An International Journal, 28(2), 205-214. doi: 10.1080/10615806.2014.931942.

Weems, C. F., Scott, B. G., Graham, R. A., Banks, D. M., Russell, J. D., Taylor, L. K., \& Marino, R. C. (2014). Fitting anxious emotion-focused intervention into the ecology of schools: Results from a test anxiety program evaluation. Prevention Science,16 (2), 200-210. doi:10.1007/s11121-014-0491-1.

Yousefi, F., \&Redzuan, M. (2010).The effect of test-anxiety on memory among Iranian high school students.International Journal of Psychological Studies, 2(1), 137-141.

Zeidner, M. (1998).Test anxiety: The state of the art. New York: Plenum Press.

Zeidner, M., \& Mathews, G. (2005).Evaluation anxiety.In A. J. Elliot, \& C. S. Dweck (Eds.), Handbook of competence and motivation (pp. 141-163).London: Guildford Press.

Recebido / Received: 22/03/2016

Aprovado / Approved: 21/10/2016 